\title{
On the Limit of Machine Intelligence
}

\author{
Jinchang Wang \\ School of Business, Richard Stockton College of New Jersey, Galloway Township, USA \\ Email: jinchang.wang@stockton.edu
}

Received September 23, 2013; revised October 20, 2013; accepted October 26, 2013

Copyright @ 2013 Jinchang Wang. This is an open access article distributed under the Creative Commons Attribution License, which permits unrestricted use, distribution, and reproduction in any medium, provided the original work is properly cited.

\begin{abstract}
Whether digital computers can eventually be as intelligent as humans has been a topic of controversy for decades. Neither side of the debate has provided solid arguments proving this way or the other. After reviewing the contentions, we show in this article that machine intelligence is not unlimited. There exists an insurmountable barrier for digital computers to achieve the full range of human intelligence. Particularly, if a robot had a human's sentience of life and death, then it would cause a logical contradiction. Therefore, a digital computer will never have the full range of human consciousness. This thesis substantiates a limit of computer intelligence and draws a line between biological humans and digital robots. It makes us rethink the issues as whether robots will remain forever one of the tools for us to use, or they will someday become a species competing with us; and whether robots can eventually dominate humans intellectually.
\end{abstract}

Keywords: Artificial Intelligence; Nature of Consciousness; Intelligent Computers

\section{Introduction}

Computer intelligence has achieved tremendous progress in the past decades. Machines are now more capable of doing the jobs requiring intelligence. This trend is continuing and accelerating. Before the first industrial revolution that was driven by mechanical machines and electricity, it was difficult for the people to imagine what a machine could do. Nowadays, amid the second Industrial revolution driven by electronic computers, it is difficult for people to imagine what a computer cannot do. People are increasingly concerned about the fate of human beings: What if a computer is as intelligent as a human? Will a computer have "mind"? Is there a limit of machine intelligence? Are we creating a species against ourselves? They are also closely related to fundamental philosophical curiosities such as who we are, where we come from, what intelligence and consciousness are, what the fundamental difference is between a machine and a human, and whether immortality is possible. It has been a long-standing debate among scientists on how far intelligent machines can go. Neither side of the contention has so far provided solid arguments of proving this way or the other.

We investigate in the article the issue that whether there is a limit for the capability of computer intelligence, and show logically that machine intelligence is not unlimited. Electronic computers will never have the full range of human consciousness and mental experience.
We prove it by using a counter-example that anxiety of death, which is a piece of human consciousness, cannot be possessed or emulated by an electronic robot.

\section{Debate on Machine Intelligence}

How intelligent a computer can eventually be has been debated for almost sixty years. Many scholars take it for grant that digital computers can achieve the full range of human intelligence and mentality and will become more intelligent than humans. The vanguards of artificial intelligence (AI) cherished an optimistic prospect of machine intelligence. Alan Turing predicted in 1950 that computers would pass the Turing Test by year 2000 [1].

Marvin Minsky, a founder of AI department in MIT, has never cast any doubt on the possibility of having computers with full human intelligence and consciousness, "Most people still believe that no machine could ever be conscious, or feel ambition, jealousy, humor, or have any other mental life-experience. To be sure, we are still far from being able to create machines that so all the things people do. But this only means that we need better theories about how thinking works.” [2]

Allen Newell and Herbert Simon at the time when AI as a subject was just set up depicted the future of computer intelligence, "There are now in the world machines that think, that learn, and that create. Moreover, their ability to do these things is going to increase rapidly until -in a visible future-the range of problems they can 
handle will be co-extensive with the range to which the human mind has been applied." [3] They thereafter extended that idea into their Physical Symbol System Hypothesis, "A physical symbol system has the necessary and sufficient means for general intelligent action." [4] They defined physical symbol system (PSS) as a physical device that contained a set of interpretable and combinable symbols and a set of processes that could operate on the symbols. A human brain and a computer are both examples of PSS. Their hypothesis states that a PSS possesses all the matters for thoughts and intelligence, and that something is intelligent if and only if it is a PSS. This idea was later known as "strong AI" [5].

An argument for strong AI is that "there is no reason to believe that biological mechanisms are inherently impossible to replicate using nonbiological materials and mechanisms.” [6] Human intelligence has remained almost unchanged for thousands of years, while computer's capability has been doubled every two years [7]. So, it is inevitable and inexorable for a computer to intellectually catch up with humans, sooner or later.

Minsky once described the human brain as a "meat machine, no more no less". "How could a device made of silicon be conscious? How could it feel pain, joy, fear, pleasure, and foreboding? It certainly seems unlikely that such exotic capacities should flourish in such an unusual silicon setting. But a moment's reflection should convince you that it is equally amazing that such capacities should show up in carbon-based meat." [8] "If we're a carbon-based complex, computational, collocation of atoms, and we're conscious, then why wouldn't the same be true for a sufficiently complex silicon-based computer?" [6]

Stephen Wolfram claimed his "thesis of software of everything" in 2002, which was even stronger than strong AI: "Beneath all the complex phenomena we see in physics there lies some simple program which, if run for long enough, would reproduce our universe in every detail.” [9]

Ray Kurzweil, a computer scientist and futurist, believes that a silicon computer can be as conscious and spiritual as a human. He is optimistic about the perspective humanoid era, taking it as a blessing for humans. In his informative and enlightening books, $<$ The age of intelligent machines>, <The age of spiritual machines>, $<$ The singularity is near $>$, and many of his articles, he argued, with seemingly irrefutable reasons, that the new era of humanoids is inexorable and near. "The human brain presumably follows the laws of physics, so it must be a machine, albeit a very complex one. Is there an inherent difference between human thinking and machine thinking? To pose the questions another way, once computers are as complex as the human brain, and can match the human brain in subtlety and complexity of thought, are we to consider them conscious? ... They (computers) will appear to have their own free will. They will claim to have spiritual experiences. And people-those still using carbon-based neurons-will believe them.” [10] Gilder and Richards commented on Kurzweil's utopia, "Kurzweil's record as a technology prophet spurred interest in this more provocative prediction that within a few decades, computers will attain a level of intelligence and consciousness both qualitatively and quantitatively beyond human capacity.” [6]

Storrs Hall, a nano-scientist and computer system architect, had no doubt that computers would soon achieve human intelligence and consciousness, and was optimistic about the "moral machines". "AI is coming. It is clear we should give conscience to our machines when we can. It also seems quite clear that we will be able to create machines that exceed us in moral as well as intellectual dimensions.” [11]

Hans Moravec, a leading expert in robotics, called for humans to give the way to the new species of intelligent machines, "We should keep researching, and should proudly work to create robots that will supplant humans as Earth's superior species. Humans should just get out of the way of this self-imposed evolution.” [12]

William. Bainbridge, as the deputy director for the Division of Information and Intelligent Systems at the National Science Foundation (NSF), predicted the possible impacts of robots on human's longevity, "in principle, and perhaps in actuality three or four decades from now, it should be possible to transfer a human personality into a robot, thereby extending the person's lifetime by the durability of the machine.” [13]

Stephen Hawking, a well-known theoretical physicist in University of Cambridge, joined the chorus in 2013 predicting that computers could have human's intelligence by "copying the brain", and "so provide a form of life after death." [14]

Some people do not believe that computers can be human-like. The strongest among them are dualists who take it for grant that the mind is something separate, and fundamentally different, from the physical things. However, they did not provide convincing arguments showing why mind was not physical, and they did not tell what mind actually was if it were not physical. AI people seemed not particularly interested in refuting dualism. "The only refutation worth doing is simply to build the AI, and then we will see who is right." [11]

As a successful and highly regarded computer architect and entrepreneur in Silicon Valley, Jeff Hawkins held a firm attitude denying the possibility of human-like computers, "Can computers be intelligent? For decades, scientists in the field of artificial intelligence have claimed that computers will be intelligent when they are powerful enough. I don't think so. ... Brains and com- 
puters do fundamentally different things.” [15]

Discretions were used by some scientists and philosophers on the future of artificial intelligence. The subtleties of a human's mind are so delicate that science seems incapable to interpret and a "mechanically" programmed computer is improbable to emulate them. John Searle, a philosopher in University of California, Berkeley, challenged the concept of machine intelligence with his "Chinese room argument", arguing "they (computers) are immensely useful devices for simulating brain process. But the simulation of mental states is no more a mental state than the simulation of an explosion is itself an explosion." He rejected the strong AI's claim that "the mind is just a computer program" [5].

Physicist and mathematician Roger Penrose of the University of Oxford enumerated in his book $<$ The emperor's new mind $>$ mysterious phenomena and processes of human mind, and said "According to this perception, all aspects of mentality are merely features of the computational activity of brain; consequently, electronic computers should also be capable of consciousness, ... I do my best to express, in a dispassionate way, my scientific reasons for disbelieving this perception, and arguing that the conscious minds can find no home within our present-day scientific world-view." He hypothesized that the thorough explanation of the human mind would be somewhere in the "quantum world". [16]

Mathematician and psychologist Douglas Hofstadter at Indiana University, Bloomington, believed that human mind would be unlikely to be programmed directly; instead, it would be an emerged phenomenon as by-products of sufficiently complex computer programs. He wrote in his Pulitzer-Prize-winning book $<$ Godel, Escher, Bach-an eternal golden braid>, "Will emotions be explicitly programmed into a machine? No. That is ridiculous. Any direct simulation of emotions cannot approach the complexity of human emotions, which raise indirectly from the organization of our minds. Programs or machines will acquire emotions in the same way: as byproducts of their structure, of the way in which they are organized—not by direct programming." [17] But he did not provide necessary or sufficient conditions for such imaginary "emergent phenomenon" to occur.

The arguments of both sides of the debate are more or less assertive, lacking strict proofs. Subtleties and mysteries of human mentality show difficulties and unlikeliness for silicon mechanisms to realize the biological mind. But unlikeliness does not amount to impossibility. On the other hand, the opponents of strong AI have failed to point out the insurmountable barriers for computers to catch up with the human intelligence given the fact that computers are becoming smarter and smarter. The limit of computer intelligence remains an open issue.

Ray Kurzweil once challenged the opponents of strong
AI to show the proofs for their assertions that non-biological things cannot be capable of what biological things are, "If one is searching for barriers to replicating brain function, it is an ingenious theory, but it fails to introduce any genuine barriers." [18]

In the next section, we tackle this open issue by showing a logically "genuine barrier" insurmountable for computer intelligence so that a robot controlled by an electronic computer will not possess full human mentality.

\section{Computer Intelligence Is Not Omnipotent}

As reviewed in the last section, whether computer programs are capable of emulating all human consciousnesses has been debated for decades among computer scientists, philosophers, physicists, mathematicians, psychologists, and other scientists. This issue is even viewed by some scholars as one that cannot be proved or disproved. We in this section reason that a digital robot is not omnipotent. A robot is not able to have the full range of human mental experience.

\subsection{Definitions of Terms}

We first define the terms to be used in this section. Meanings of those daily-used words need to be specified in an exact way before we use them in reasoning.

Consciousness in this article refers to all mental phenomena of a person such as thinking, calculating, reasoning, feelings, emotions, intuitions, and faith. Andy Clark categorized mental phenomena of "consciousness" with three levels [8]:

1) The feelings that characterize daily experience (hunger, sadness, desire, ...)

2) The flow of thoughts and reasons;

3) The meta-flow of thoughts about thoughts, thoughts about feelings, and reflection on reasons.

Although lower level consciousnesses are observed in all animals, the high level ones such as awareness of self and thoughts-about-thoughts are associated only with human beings.

A computer program, or simply a program, refers to a set of instructions to the computer in a computer language. By robot we refer to a machine under control of its internal digital computer, which is able to move and act like a human.

A program is copiable or duplicatable if the instructions in the program can be duplicated so that the original and the copy are literally identical and the result of running the copy is indistinguishable from the result of running the original. With this definition, once a computer is able to do square-root calculation, for example, its program can be copied to other computers so that they all can calculate square-root, and one cannot tell that the 
result of square-root of 3.76, for example, is from running the original program or from running its copy. Once a computer is able to do spelling-check, other computers, by copying, can do the same exactly. With this definition, once a computer will someday be programmed to have consciousnesses of "happiness", "self-awareness" and "anxiety of death", other computers, by copying, will also have same consciousnesses of "happiness", "selfawareness" and "anxiety of death".

\subsection{A Digital Computer Is Copiable}

A program in a digital computer is copiable. That is because: i) A program for a digital computer is a step-bystep procedure or algorithm; 2) Any algorithmic procedure, according to Church-Turing Thesis $[16,17,19]$, can be converted to a set of equivalent $0-1$ codes for a Turing Machine; 3) The 0 - 1 codes on the tape of a Turing Machine are obviously copiable.

If all the programs in a computer are copiable, then we say that the computer is copiable. All computers we have had so far are copiable since all programs in a digital computer are copiable.

\subsection{Examples of Human Consciousnesses}

Self-awareness is a conscious trait "associated with the tendency to reflect on or think about one-self” [20]. Selfawareness is a part of intelligence that differentiates subjective selfhood from the other beings. It belongs to the third level of consciousness in Clark's classification (see Section 3.1). A human is capable of reflecting on his own mental experience and recognizing self-identity, while the other animals are not.

Death is the destination of life. Death will belong to everyone with no exception. No matter how hard one tries to forget it and avoid it, it will come anyway. Once one had it, s/he would have it forever. No one knows exactly what it is like after death. Anxiety is "an emotion of feeling dominated by comprehensions" [21]. Anxiety of death is comprehension and dread of the mystery and obscurity of death. The feeling of anxiety of death is due to the intelligence of a human, realizing that "I live only once" and "if I died then the world currently around me would disappear forever."

People may disagree on the exact definition of "consciousness". But they would agree that self-awareness and anxiety of death are two examples of human consciousnesses, which is sufficient for the purpose of addressing our thesis in this article.

\subsection{Anxiety of Death Defies Copying}

Let $A D$ denote "anxiety of death", and $S A$ denote "selfawareness". Let $R$ denote a digital robot. Suppose that $\mathrm{R}$ is programmed to have all human consciousnesses. A human has consciousnesses of $\mathrm{AD}$ and $\mathrm{SA}$, so does robot R.

Suppose all the programmed consciousnesses in robot $\mathrm{R}$, including SA and $\mathrm{AD}$, are copied to another robot R'. According to the definition of "copying" in Section 3.1, $\mathrm{R}$ and $\mathrm{R}$ ' have identical consciousnesses after copying, which include self-consciousness and self-identity. That is, the self-identities of R and R' are same. $R$ and R' are a same "self", which can be put as R-self = R'-self. Now, either R or R' has multiple "self's". Realizing this, R would not fear to die since "death" of itself would not result in disappearance of the world around itself due to the existence of R'-self that is another R-self. Therefore, $\mathrm{R}$ would not have anxiety of death. By the same token, robot R' would not have anxiety of death either.

Hence, the outcome of copying R to R' is: The copy $R^{\prime}$ does not have $A D$ as supposed, and the original $R$ loses AD. Such "copying" is not the "copying" as we defined in 3.1 since the original is not completely duplicated and this "coping" changes the original. In other words, if $\mathrm{AD}$ is copied, then $\mathrm{AD}$ is lost. Therefore, we say that $A D$ defies copying.

\subsection{A Robot Will Never Have Full Range Consciousness of Humans}

Suppose that a digit robot $\mathrm{R}$ has been programmed to have all intelligence and consciousness as a biological human does. So, R has SA and AD, same as a human. Robot $\mathrm{R}$ is free in moving and acting same as a human is, so as to maintain those human consciousnesses related to moving and actions.

As discussed in Section 3.4, the programmed consciousness $\mathrm{AD}$ in $\mathrm{R}$ is copy-defiant so that if $\mathrm{AD}$ is copied together with $\mathrm{SA}$ to another robot, then $\mathrm{AD}$ would be lost from both the original and the copy. So, the programs for $\mathrm{AD}$ and $\mathrm{SA}$ in robot $\mathrm{R}$ must have no copy in other robots to maintain the existence of $\mathrm{AD}$. Since $\mathrm{R}$ is as intelligent as humans, $\mathrm{R}$ would be able to figure out that "a copy of the programs in me would relieve my anxiety of death". So, $\mathrm{R}$ would have a motive to make a copy of the programs of its consciousnesses.

If $\mathrm{R}$ managed to get itself copied, then $\mathrm{AD}$ would no longer exist with $\mathrm{R}$ since $\mathrm{AD}$ defies copying. But humans still have $\mathrm{AD}$. So, robot $\mathrm{R}$ would have different consciousness from humans at least on the feelings towards death. Note that R should easily get itself copied because $\mathrm{R}$ is free in action, and copying computer programs is a simple routine of computer operation: -duplicating all the programs inside $\mathrm{R}$ would be as easy as making a backup of all the files in a computer.

If $\mathrm{R}$ did not get itself copied for some reason, then $\mathrm{R}$ would still have $\mathrm{AD}$ as initially it had. But robot $\mathrm{R}$ would have a motive to have a copy of itself to relieve $A D$, and $\mathrm{R}$ would know that it could be done as easy as doing 
"copy-paste" at a computer. On the other hand, although a biological human also has a motive to relieve AD by making himself copied, he realizes that copying himself is very difficult or almost impossible. Now, $\mathrm{R}$ has $\mathrm{AD}$ but knows that its "death" can be easily avoided by making himself copied; while a human has no idea on how to avoid death. For robot R, death can be avoided with a simple process of program-copying. For the human, on the other hand, death is the inexorable destination. So, robot $\mathrm{R}$ and a human would inherently have different feelings towards death. That difference is analogical to the feeling of a man who has caught a slight cold that can be cured easily versus the feeling of a man who has got a terminal cancer that is past beyond cure.

Thus, robot $\mathrm{R}$ will not have same consciousnesses as a human does, no matter whether or not R has a copy of its consciousnesses: -If $\mathrm{R}$ got itself copied, robot $\mathrm{R}$ would not have AD but the human still has; If R did not get itself copied, $\mathrm{R}$ would have a feeling toward death different from the human. It contradicts to the assumption that $\mathrm{R}$ has been programmed to have all consciousnesses as a biological human has. In other words, the assumption that digit robot $\mathrm{R}$ has all human's consciousnesses is not logically valid. Therefore, a digit robot controlled by an electronic computer cannot be programmed to have the full range of human consciousness, since a robot would not have, at least, the sentience towards "death" as a human does.

The above arguments have an implicit assumption: we are not able to duplicate an existing person's "self" by any technology such as programming and cloning. So, robot $\mathrm{R}$ does not have the self-consciousness of anyone existing in the world. This assumption ensures anxiety of death (AD) remains with the human since a human's self is not programmed or copied to either a robot $\mathrm{R}$ or some other objects. If a person knew how to copy himself then he would lose the sentience of AD due to the existence of his "copies". Copying "self" has been a dream of human beings, but it has not yet come true. "Cloning" is a step toward realizing the dream, but it is, at least for now, not "copying self”. Cloning a sheep is to duplicate a sheep so that the copy looks identical to the original. But the "self" of the cloned sheep, if it could sense it, is not the original one. Imagine that one day one would be able to clone a human. The original person would not agree to be destroyed after being cloned, because he knew the cloned copies were not "himself"!

Therefore, a robot cannot have human's sentience of death and living, which will hold true at least till the time when humans come to know how to duplicate themselves. The thesis below summarizes what we have derived:

Thesis-1:

A robot controlled by an electronic computer will not have all human consciousnesses, and so will not have the same mental experience as a human, as far as we do not know how to duplicate an existing person's self-consciousness.

\subsection{Summary of the Arguments}

The arguments for showing Thesis- 1 addressed in sections 3.2 through 3.5 are briefed as follows:

a) Anxiety of death is a part of consciousness of humans.

b) Anxiety of death defies copying.

c) Electronic computers are copiable.

d) Robots controlled by electronic computers cannot have anxiety of death. (Due to b) and c))

e) Robots controlled by electronic computers cannot have the full range of human consciousness. (Due to a) and d))

Therefore, machine intelligence is not unlimited. "Anxiety of death" is a piece of human consciousness which is unachievable for computer intelligence.

\section{Implications and Discussions}

Kurzweil made a prediction in his 2005 book, "By the late 2020s, we will have completed the reverse engineering of the human brain, which will enable us to create nonbiological systems that match and exceed the complexity and subtlety of humans, including our emotional intelligence.” [22] Stephen Hawking predicted in September 2013 that "It's theoretically possible to copy the brain on to a computer and so provide a form of life after death." [14] Thesis-1 in this article shows that the above predictions will not come true. It is logically impossible that electronic computers and robots have our human's full experience of consciousness and mentality through either programming or copying, even programming or copying some of human consciousnesses is not impossible.

"Anything is not impossible, unless it causes a logical contradiction" (Gottfried Leibniz). It is the feature of "duplicatability" of computer programs that would cause a logical contradiction if a computer had the consciousness of anxiety of death. All digital computers are copiable as reasoned in Section 3.2. All man-made machines we have had so far are copiable. By Thesis-1, machines will not be as conscious as humans no matter how complex machines are as far as they are copiable. In other words, machines will not be as conscious as humans unless they are not copiable. Up to now, humans have not made an un-copiable machine. How to construct an uncopiable machine is still beyond our knowledge at this time.

Emulating human intelligence on computers has been an ultimate quest of artificial intelligence (AI). However, “today's AI bears little resemblance to its initial concep- 
tion,” [23]. Rather than pursuing re-creating human intelligence, today's AI tries to use the accomplishments of AI so far, combined with Internet, to master discrete tasks, such as manipulating stock market, automatic driving, Internet searching, and fraud detection [24]. This redirection of AI does not signal the abandonment of AI's original quest of emulating human intelligence. It signals the difficulties of that quest. Emulating human intelligence on a machine seems a goal farther than people initially think, though it remains as the goal for AI people.

We humbly admit that we are very ignorant about our own consciousness, motions, mind, mentality, spirit, and soul. Is there any piece of our consciousnesses, other than anxiety of death that may not be emulated by an electronic computer? Can an existing person's mind be programmed? Can a human's "self" be copied? What is an un-copiable machine like, and how does it work? Is consciousness a "by-product" emerging from sufficiently sophisticate programs, as proposed by Hofstadter [17]? If so, how does such "emerging" process occur? Is the emerged consciousness copiable? These are examples of the issues for us to keep reflecting hereafter.

\section{REFERENCES}

[1] A. Turing, "Computing Machinery and Intelligence," Mind, Vol. 59, 1950, pp. 433-466. http://dx.doi.org/10.1093/mind/LIX.236.433

[2] M. Minsky, "The Society of Mind," Touchstone, Simon \& Schuster, New York, 1986, p. 19.

[3] A. Newell and H. Simon, "Heuristic Problem-Solving: The Next Advance in Operation Research," Operations Research, Vol. 6, No. 6, 1958.

[4] A. Newell and H. Simon, "Computer Science as Empirical Inquiry: Symbols and Search," Communications of the Association for Computing Machinery, Vol. 19, No. 3, 1976, pp. 113-126. http://dx.doi.org/10.1145/360018.360022

[5] J. Searle, "The Mystery of Consciousness," The New York Review of Books, New York, 1997, p. 14.

[6] G. Gilder and J. Richards, "Are We Spiritual Machines? The Beginning of Debate,” In: J. Richards, Ed., Are We Spiritual Machine? Ray Kurzweil vs. the Critics of Strong AI, Discovery Institute Press, Seattle, 2002, p. 11.

[7] G. E. Moore, "Cramming More Components onto Integrated Circuits,” Electronics, Vol. 38, No. 8, 1965

[8] A. Clark, "Mindware: In Introduction to the Philosophy of Cognitive Science," Oxford University Press, New York, 2001, p. 2.
[9] S. Wolfram, “A New Kind of Science,” Wolfram Media, Inc., Champaign, 2002, p. 35.

[10] R. Kurzweil, "The Age of Spiritual Machines-When Computers Exceed Human Intelligence,” Penguin Books, Middlesex, New York, 1999, pp. 5-6.

[11] J. Storrs Hall, "Beyond AI: Creating the Conscience of the Machine,” Prometheus Books, New York, 2007, p. 367.

[12] H. Moravec, “Bill Joy’s Hi-Tech Warning,” 2001. http://www.gigablast.com/get?q=\&c=dmoz3\&d=1180344 $53864 \&$ cnsp $=0$

[13] W. S. Bainbridge, "Progress toward Cyberimmortality," In: I. Basset, Ed., The Scientific Conquest of Death: Essays on Infinite Lifespans, Immortality Institute, Wausau, 2004, p. 117.

[14] M. Bennett-Smith, "Stephen Hawking: Brains Could Be Copied To Computers To Allow Life After Death,” The Hoffington Post, Science, 2013. http://www.huffingtonpost.com/2013/09/24/stephen-haw king-brains-copied-life-after-death_n_3977682.html

[15] J. Hawkins and S. Blakeslee, “On Intelligence,” Holt Paperback, Times Books, Henry Holt and Company, New York, 2004, p. 5.

[16] R. Penrose, “The Emperor's New Mind,” Oxford University Press, Oxford, 1999, pp. 61-64.

[17] D. R. Hofstadter, “Godel, Escher, Bach: An Eternal Golden Braid,” Basic Books, Inc., New York, 1999, pp. 676677.

[18] R. Kurzweil, "The Evolution of Mind in the Twenty-First Century,” In: J. Richard, Ed., Are We Spiritual Machine? Discovery Institute Press, New York, 2002, p. 48.

[19] S. Russell and P. Norvig, "Artificial Intelligence-A Modern Approach,” 3rd Edition, Prentice Hall, New Jersey, 2010, p. 8.

[20] “Encyclopedia of Psychology," Oxford University Press, Oxford, Vol. 7, 2000, p. 209.

[21] J. A. Popplestone and M. W. McPherson, "Dictionary of Concepts in General Psychology,” Greenwood Press, New York, 1988, p. 21.

[22] R. Kurzweil, "The Singularity Is Near-When Humans Transcend Biology,” Penguin Books, New York, 2005, p. 377.

[23] Steven Levy, “The A.I. Revolution,” Wired, Vol. 19, No. 1, 2011, pp. 86-89.

[24] F. Salmon and J. Stokes, "Bull vs. Bear vs. Bot," Wired, Vol. 19, No. 1, 2011, pp. 90-93. 